\title{
A PROSPECTIVE RANDOMIZED DOUBLE BLIND PLACEBO CONTROLLED CROSSOVER STUDY OF FLUOXETINE EFFICACY IN THE PROPHYLAXIS OF CHRONIC DAILY HEADACHE IN CHILDREN AND ADOLESCENTS
}

\author{
José Luiz Dias Gherpelli', Sandro Blasi Esposito'
}

\begin{abstract}
Thirty-two children (21 female and 11 male), between 7 and 14 years old, with chronic daily headache $(\mathrm{CDH})$ were consecutively included in a prospective, randomized, double blind, placebo controlled crossover study. The patients were divided in group I (fluoxetine vs. placebo), with 17 patients and g roup II (placebo vs. fluoxetine), with 15 patients. After one month of baseline headache frequency recording, the patients received fluoxetine in dosages from 0.25 to $0.50 \mathrm{mg} / \mathrm{kg}$ for three months. A wash out period of one month was followed by another three months treatment period. Results showed a significant decrease in headache frequency in the study period [78\% reduction in group I $(p<0.025)$, and $45 \%$ reduction in group II $(p=0.025)]$. Gastrointestinal adverse effects were observed in nine patients $(29 \%)$ that received fluoxetine, compared with $3(10 \%)$, with placebo. We conclude that fluoxetine efficacy is not higher than placebo in the prophylaxis of CDH in children and adolescents.
\end{abstract}

KEY WORDS: headache, chronic daily headache, children and adolescents, migraine, fluoxetine.

\begin{abstract}
Estudo randomizado prospectivo duplo-cego cruzado controlado com placebo da eficácia da fluoxetina na profilaxia da cefaléia crônica diária na infância e adolescência

RESUMO - Trinta e duas crianças (21 feminino, 11 masculino), com idades entre 7 e 14 anos, com cefaléia crônica diária (CCD) foram incluídos consecutivamente no estudo prospectivo, randomizado, duplo-cego, c ruzado com placebo. Os pacientes foram divididos em grupo I (fluoxetina vs. placebo), com 17 pacientes, e grupo II (placebo vs. fluoxetina) , com 15 pacientes. Após um mês de registro da freqüência da cefaléia, os pacientes receberam fluoxetina em doses de 0,25-0,5 mg/kg por três meses. Após período de interrupção da droga de um mês, seguiu-se novo período de três meses de tratamento. Os resultados mostraram diminuição estatisticamente significativa da freqüência da cefaléia $[78 \%$ de redução no grupo I $(p<0,025)$ e $45 \%$ de redução no grupo II $(p<0,025)$ ]. Efeitos colaterais gastrointestinais ocorreram em 9 pacientes (29\%) na vigência da fluoxetina, comparado com 3 (10\%) com placebo. Concluímos que não houve diferença entre o efeito da fluoxetina e do placebo na profilaxia da CCD em crianças e adolescentes.
\end{abstract}

PALAVRAS-CHAVE: cefaléia, cefaléia crônica diária, crianças e adolescentes, migrânea, fluoxetina.

The term chronic daily headache $(\mathrm{CDH})$ is used to refer to headaches that occur $\geq 15$ days/month. Silberstein et al. ${ }^{1}$ classified $\mathrm{CDH}$ into two types, each with or without medication overuse. CDH is divided into primary and secondary varieties. Primary CDH can be subclassified into disorders of short ( $<4$ hours) and long ( $>4$ hours) duration. Primary $\mathrm{CDH}$ of long duration include chronic tension-type headache, chronic migraine (previously called transformed migraine), new daily persist- ent headache and hemicrania continua 1 . In adults, $4-5 \%$ of the general population have $\mathrm{CDH}^{2}$. There are few studies addressing $\mathrm{CDH}$ in children. Almost $50 \%$ of children with frequent headaches are left without diagnosis, when the adult diagnostic criteria are used in this age group, what lead to the proposal of a new CDH category for children called comorbid or mixed pattern, that includes cases in which migrainous and tension-type headache patterns exist independently of each other, without

Child and Adolescence Headache Clinic, Department of Neurology, Clinics Hospital, University of São Paulo Medical School, São Paulo SP, Brazil; ${ }^{1} \mathrm{MD}$, PhD Pediatric Neurologist. Financial support: Sigma-Pharma Company.

Received 8 December 2004, received in final form 3 March 2005. Accepted 15 April 2005.

Dr. José Luiz D. Gherpelli - Avenida Albert Einstein 627 / 13º floor / Suite 1308 - 05651-901 São Paulo SP - Brasil. E-mail: jldg@osite.com.br 
any transformation ${ }^{3,4}$. Abu-Arafeh ${ }^{5}$ studied 115 children and adolescents with almost daily headache and found that $63.5 \%$ had chronic tensiontype headaches, while $30.4 \%$ had independent episodes of migrainous and tension-type headaches, the comorbid type, and only $4.3 \%$ were classified as analgesic overuse headache. Among 40 children and adolescents with chronic daily headaches over the last 6 months, we found $65 \%$ of transformed migraine, $17.5 \%$ of the mixed pattern and $17.5 \%$ of chronic tension-type headache ${ }^{6}$. The treatment of primary CDH is complicated by analgesic overuse, comorbid psychiatric disorders, low frustration tolerance and emotional problems in adults. There are few well-designed studies in the literature, although the antidepressant amitriptyline and several drugs used for migraine prophylaxis seem to be useful'2.

Fluoxetine, a selective serotonin-reuptake inhibitor antidepressant, is a phenylpropylamine-derivative. The drug differs structurally from other selective sero to $\mathrm{n}$ in - reup take inhibitor antidepre ss a $\mathrm{n}$ ts (e.g., citalopram, paroxetine, sertraline) and also differs structurally and pharmacologically from other currently available antidepressant agents (e.g., tricyclic antidepressants, monoamine oxidase inhibitors). Fluoxetine is widely used as antidepressive in adults, as well as in children and adolescents $^{7}$. Efficacy of fluoxetine for migraine prophylaxis was reported by some authors ${ }^{8,9}$, while others were unable to observe such effect ${ }^{10}$. A doubleblind placebo-controlled study showed fluoxetine to be useful in the treatment of chronic daily headache ${ }^{11}$. Others, using it in association with amitriptyline, could not observe this effect ${ }^{12}$.

The objective of this study was to test the eff icacy of fluoxetine prophylaxis in a group of chil$\mathrm{d}$ ren and adolescents with primary $\mathrm{CDH}$ through a prospective, randomized, double blind, placebo-controlled, crossover study.

\section{METHOD}

F rom January 1999 to April 2002, 40 children (30 female and 10 male), between 7 and 14 years old, with a diagnosis of primary $\mathrm{CDH}$ according to the Silberstein criteria were seen in the outpatient headache clinic. Thirty-two patients ( 21 female and 11 male) agreed to participate in our study. The ethics commission of our institution previously approved the study protocol and a signed informed consent was obtained from the parents.

All patients had a normal neurological examination and were instructed to fill out questionnaires of selfevaluation for depression, anxiety and life quality.
None of the patients used common analgesics more than 10 days/month during the study period. Patients were allowed to take the analgesics that were currently used before the beginning of the study. No patient used analgesics containing narcotics. The authors did not change the type of analgesic that was being used before the study period. Only the dosage was adjusted according to the patient's age and weight. The most commonly used were paracetamol and dipirone, with or without caffeine, isomeptene, and ergotamine. Analgesics for acute pain relief were allowed during the wash-out period as well.

Environmental factors were not controlled during the study period, but no specific advices were given conce rning daily activities, sleep or food habits, to the patients or their families.

All patients had a primary headache with a headache $f$ requency of $\geq 15$ days/month, for a period of at least 6 months before the initial evaluation. $\mathrm{CDH}$ diagnosis was based on Silberstein et al. ${ }^{1}$ and Gladstein and Holden ${ }^{3}$ modified criteria for children. Chronic daily migraine (tran sforme d migraine) included meeting Intern a ti o n a I Headache Society (IHS) criteria for migraine in the past, daily or almost daily (>15 days/month) headache for more than 1 month, average headache duration of 4 hours, and a history of increased headache frequency with decreased migraine symptoms over the last 3 months ${ }^{1}$. Criteria for chronic tension-type headache included headache frequency of more than 15 days per month, with a duration of pain lasting more than 4 hours per day for at least 6 months. Also, patients should have pressing, bilateral pain with mild to moderate severity that is not aggravated by physical activity. There should be a history of episodic tension-type headache in the past, with an evolution to daily headaches in the past 3 months, and an absence of autonomic symptoms ${ }^{1}$. Mixed (or comorbid) pattern included patients with a high frequency of migraine headache symptomatology interm ixed with tension type headache ${ }^{3}$.

The patients were randomly assigned to two groups: group I received fluoxetine and later placebo, and group II that started with placebo and received fluoxetine in the last study period. After one month of baseline headache frequency re c o rding, the patients received fluoxetine (or placebo) in dosages that varied from 0.25 to $0.50 \mathrm{mg} / \mathrm{kg}$ for three months. A wash out period of one month was followed by another three months period of drug treatment (Fig 1).

The drug were delivered in solution flasks of $50 \mathrm{ml}$ (10 $\mathrm{mg} / \mathrm{ml}$ of fluoxetine) labeled with the letters $A$ or B. The initial fluoxetine dosage was $0.25 \mathrm{mg} / \mathrm{kg} / \mathrm{day}$, administered once a day, in the morning, and was eventually increased up to a maximum dosage of $0.5 \mathrm{mg} / \mathrm{kg} /$ day, or $20 \mathrm{mg} / \mathrm{day}$, according to the therapeutic response (reduction $<50 \%$ of headache frequency).

Monthly visits were scheduled where headache frequency and adverse effects were recorded through a 


\section{Group I}

Group II

1 Month Baseline

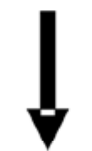

3 Months Fluoxetine

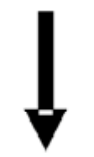

1 Month Wash Out

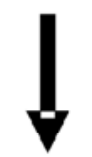

3 Months Placebo
1 Month Baseline

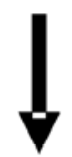

3 Months Placebo

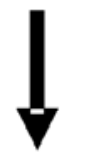

1 Month Wash Out

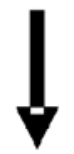

3 Months Fluoxetine

Fig 1. Design of the study.

headache diary. The presence and intensity of adverse effects lead to dosage decrease, or drug withdrawal.

Drug compliance was monitored through the flasks residual volume, after each visit.

The following tests were used for statistical analysis: 1 . Friedman test for rank variance analysis, with the objective of comparing headache frequency, for each patient, in each visit, and, when significant, was complemented by the test of multiple comparisons. 2. Wilcoxon test comparing the baseline and final headache frequency for each patient. Significance value was $p<.05$. The test power was established at $80 \%$, for a decrease in headache frequency of $\geq 50 \%$.

\section{RESULTS}

Group I included 17 patients ( 11 female, 6 male) and group II, 15 patients ( 10 female, 5 male). Of the 17 patients initially included in group I, 7 completed the whole study, 4 completed only the first th ree months period, and 6 abandoned the study

for several reasons ( 2 for lack of response, 3, due to adverse effects, and one moved to another state). In group II, of the 15 initially randomized patients, 10 completed the study, 2 completed only the first three months period, and 3 dropped out (one due to adverse effects and 2 for ignored reasons).

Seventeen patients completed the whole study period, 7 in group I, and 10, in group II, and data on these patients were used for statistical analysis purposes. The sex distribution, mean age, initial and final headache frequency, and CDH diagnosis are shown in the Table. Seventy percent of the patients were classified as chronic daily migraine (transformed migraine), $23.5 \%$, as mixed (comorbid) pattern, and only one patient, as chronic tension-type headache.

There was no statistically significant difference between the children that completed the study and the dropouts concerning age, sex, headache frequency, analgesic intake, headache diagnosis, and questionnaires ranks of anxiety, depression and life quality.

Six of the seventeen patients that completed the study (35.3\%) had scores that suggested a depressive trait, two patients of group I, and 4, of group II. Four (23.5\%) patients had scores that revealed a high anxiety status, one of group I, and 3 , of group II. Four patients (23.5\%) had low scores in the quality of life questionnaire, one of group I, and 3, of group II.

Headache frequency during the study period is shown in Fig 2.

The mean monthly headache frequency of group I (fluoxetine) decreased from 18.8 to 5.7 (70\% reduction), and of group II (placebo), from 23.9 to 14.2 (40\% reduction), during the first three months period. Statistical analysis using Friedman variance test for ranks showed a significant decrease in headache frequency in both group I $(p<0.02)$ and group II $(p<0.02)$ after the first three months period (Fig 3).

Table. General data of the groups that completed the study.

\begin{tabular}{|c|c|c|c|c|}
\hline Group & Sex & Age (mean) & $\begin{array}{l}\text { Initial / Final mean } \\
\text { headache frequency }\end{array}$ & $\mathrm{CDH}$ diagnosis \\
\hline 1 & $6 \mathrm{~F} / 1 \mathrm{M}$ & 9.7 y $(8-12)$ & $18.5 / 4.1$ & $6 \mathrm{CDM} ; 1 \mathrm{MP}$ \\
\hline II & $6 \mathrm{~F} / 4 \mathrm{M}$ & 11.4 y $(7-14)$ & $23.9 / 13.1$ & $6 \mathrm{CDM} ; 3 \mathrm{MP} ; 1 \mathrm{CTTH}$ \\
\hline
\end{tabular}




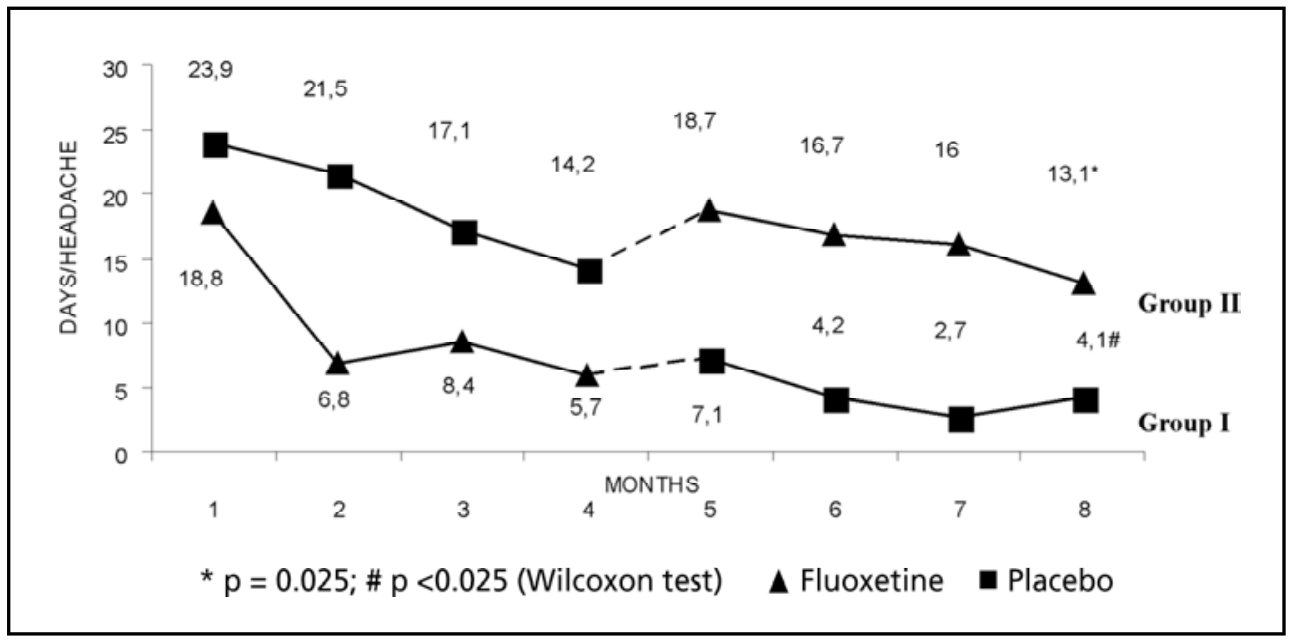

Fig 2. Mean monthly hea dache frequency during the study period.

Fig 3. Variance for ranks in the study groups.

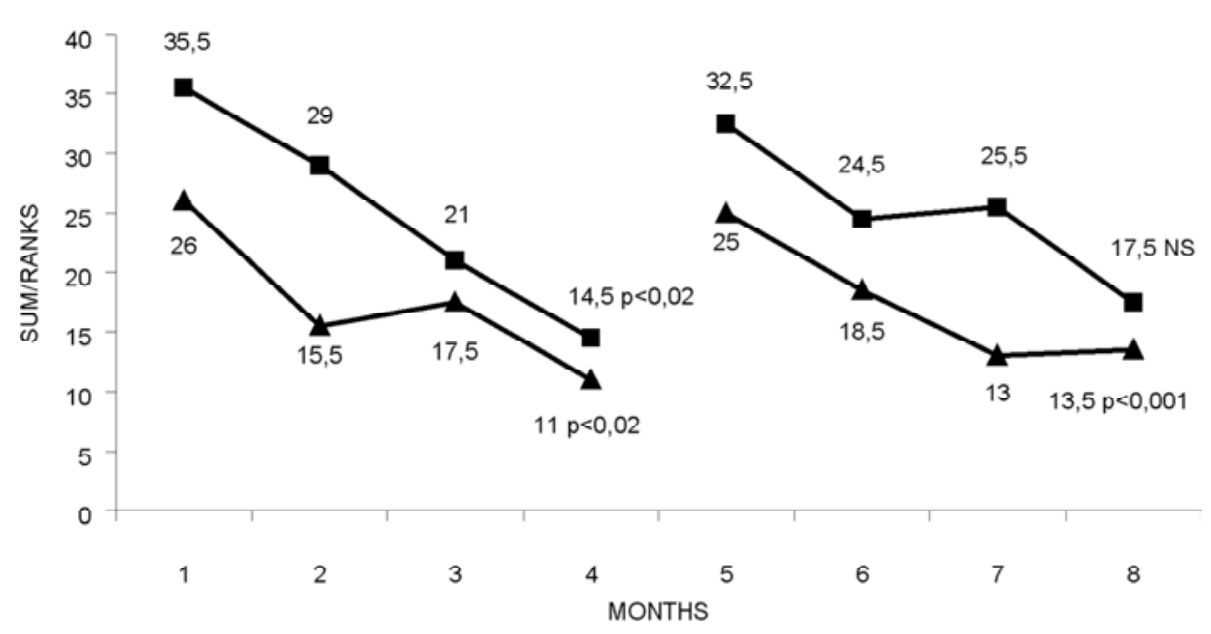

A Fluoxetine - Placebo (Group I)
Placebo - Fluoxetine (Group II)

NS, non significant
In the last three months period the mean monthly headache frequency decreased from 7.1 to 4.1 ( $42 \%$ reduction), in group I, while in group II, the frequency decreased from 18.7 to 13.1 (30\% reduction), as shown in Fig 2 . In the second study period, we only observed a significant decrease of the monthly headache frequency in patients receiving placebo $(p<0.001)$. There was no significant decre ase in headache frequency in patients taking fluoxetine (Fig 2).

We observed that both groups had a significant decrease of headache frequency when we compared the initial baseline frequency with that recorded at the end of the seven months of the study period. Patients in group I had a decrease of mean monthly headache frequency from 18.8 to 4.1 ( $p<0.025)$, a $78 \%$ reduction, and patients in g roup II, from 23.9 to 13.1 ( $p=0.025)$, a $45 \%$ reduction, as shown in Fig 2.

Thirty-one patients received at least one month of fluoxetine and 29, of placebo. Nine patients $(29 \%)$ referred adverse effects possibly related to fluoxetine, and four had the drug withdrawn and could not complete the study. Three patients (10\%) receiving placebo complained of adverse effects. Gastrointestinal system complaints (nausea, diarrhea) were the only adverse effects recorded.

\section{DISCUSSION}

Considering the whole study period (seven months), our study showed a statistically significant decrease in headache frequency that was independent of the drug. The reduction was statistically significant for patients taking either flu- 
oxetine, or placebo, in the first three months, and only for patients on placebo, in the last three months. The overall reduction, considering the 17 patients that completed the whole study period, was of $60 \%$ of the initial headache frequency. These results indicate that $\mathrm{CDH}$ in children and adolescents has a natural history that is more benign, in terms of spontaneous decrease of headache frequency, from that observed in adults.

The placebo effect observed in our study was of $40 \%$ reduction in headache frequency in both parts of the study, a higher rate than that observed by others in adults, around $25-30 \%{ }^{10,11}$.

Although several patients exhibited psychological traits suggestive of depression and anxiety, we could not establish their role in headache frequency variation due to the small sample size.

The low number of patients that completed the study could be a confounding factor in the inter$p$ retation of the results. A high drop out rate with studies using this methodology and the long treatment period is a problem frequently observed. We a g ree that a higher number of patients would be desirable. Notwithstanding, when performing studies looking for higher than $40 \%$ effectiveness in symptom's reduction, together with long study periods, the number of patients needed for valid statistical purposes is considerably reduced, and our data is within the values required ${ }^{13}$. We tried to design the study according to recent recommendation of the IHS for the study of drug efficacy in headache patients ${ }^{14}$, and we could not find any other study in the literature that used this strict methodology in children and adolescents.

We conclude that fluoxetine is not superior to placebo in the treatment of $\mathrm{CDH}$ in children and adolescents. $\mathrm{CDH}$ in this age group may have a dif- ferent natural history from that observed in adults. The high rate of spontaneous reduction of headache frequency over several months observed in our study is a fact that should lead to other studies that address the basic mechanisms for $\mathrm{CDH}$ maintenance in children. This should be taken into account when studying $\mathrm{CDH}$ in this age group.

Acknowledgments - Appreciation is extended to Dr. Neil Ferreira Novo for statistical analysis.

\section{REFERENCES}

1. Silberstein SD, Lipton RB, Solomon S, Mathew NT. Classification of daily and near daily headaches: proposed revisions to the IHS classification. Headache 1994;34:1-7.

2. Silberstein SB, Lipton RB. Chronic daily headaches. Curr Opin Neurol 2000;13:277-283.

3. Gladstein J, Holden EW. Chronic daily headache in children and adolescents: a 2 year prospective study. Headache 1996;36:349-351.

4. Gladstein J, Holden EW, Winner P, Linder S. Chronic daily headache in children and adolescents: current status and recommendations for the future. Headache 1997;37:626-629.

5. Abu-Arafeh I. Chronic tension-type headache in children and adolescents. Cephalalgia 2001;21:830-836.

6. Esposito SB, Gherpelli JLD. Chronic daily headaches in children and adolescents: a study of clinical characteristics. Cephalalgia 2004;24: 476-482.

7. Messiha FS. Fluoxetine: a spectrum of clinical applications and postulates of underlying mechanisms. Neurosci Biobehav Rev 1993;17: 385-396.

8. D'Amato CC, Pizza V, Marmolo T, Giordano E, Alfano V, Nasta A. Fluoxetine for migraine prophylaxis: a double-blind trial. Headache 1999;39:716-719.

9. Adly C, Straumanis J, Chesson A. Fluoxetine prophylaxis of migraine. Headache 1992;32:101-104.

10. Saper JR. Silberstein SD, Lake AE III, Winters ME. Double-blind trial of fluoxetine: chronic daily headache and migraine. Headache 1994;34:497-502.

11. Fontes-Ribeiro CA. I-5-hydroxytryptophan in the prophylaxis of chronic tension-type headache: a double-blind randomized placebo-controlled study. Cephalalgia 1999;19:453.

12. Krymchantowski AV, Silva MT, Barbosa JS, Alves LA.Amitriptyline versus amitriptyline combined with fluoxetine in the preventive treatment of transformed migraine: a double-blind study. Headache 2002; 42:510-514.

13. Tfelt-Hansen P, Nielsen SL. Patients numbers needed in prophylactic migraine trials. Neuroepidemiology 1987;6:214-219.

14. International Headache Society. Guidelines for controlled trials of drugs in migraine and tension-type headache. Member's Handbook. Stockholm: Scandinavian University Press, 1998:109-159. 